\title{
Minmax and Maxmin of Repeated Games with Incomplete Information
}

\author{
By J.-F. Mertens, Louvain ${ }^{1}$ ), and S. Zamir, Jerusalem ${ }^{2}$ )
}

\begin{abstract}
For a class of 2-Person 0-sum repeated games with incomplete information, Aumann/Maschler [1967] and Stearns [1967] have given a necessary and sufficient condition for the existence of $v_{\infty}$ (the value of the infinitely repeated game). Mertens/Zamir [1971] and Mertens [1971/72] have given the formula (and thus proved the existence) of $\lim _{n \rightarrow \infty} v_{n}$, the limit of the values of the games with $n$ repetitions, for a much larger class of games than that treated by Aumann/Maschler and Stearns. In this paper we extend the Aumann-Maschler-Stearns results to the larger family of games studied by Mertens [1971/72].
\end{abstract}

\section{Introduction}

We consider a two-perșon zero-sum game in which the payoff matrix depends on the "state of nature" which is an element of a finite set of states $K$. The state of nature is chosen by chance according to a given probability distribution on $K$. Each player gets some partial information as to what is the actual state of nature chosen. With this initial information, the two players start playing the game repeatedly $n$ times or $\infty$ many times. Along the play of the game some information is revealed to each player separately. This information is determined in a specified way by the moves of the players which may depend on their information on the state of nature. Thus this information flow may enable a player to learn more about the other player's knowledge on the state of nature. We will be interested in a kind of value for such infinitely repeated games.

To specify the contribution of the present paper in this general framework let us first define the model formally.

\section{The Model}

The games we consider consist of the following elements:

$K=\{1, \ldots, k\}$, a finite set. (The set of states of nature.)

$p=\left(p^{1}, \ldots, p^{k}\right)$, a probability distribution on $K$.

\footnotetext{
1) Prof. Jean-François Mertens, Center for Operations Research and Econometrics, University Catholique de Louvain, Core, 34 Voie du Roman Pays, Belgium.

2) Shmuel Zamir, Statistics Dept., The Hebrew University, Jerusalem, Israel.
} 
$K^{\mathrm{l}}=\left\{K_{1}^{\mathrm{I}}, \ldots, K_{\mu}^{\mathrm{I}}\right\}$ and $K^{\mathrm{Il}}=\left\{K_{1}^{\mathrm{HI}}, \ldots, K_{\nu}^{\mathrm{II}}\right\}$, two partitions of $K$.

$A^{r}=\left(a_{i j}^{r}\right)$ for each $r \in K$, are $m \times l$ payoff matrices.

$H^{1}$ and $H^{\mathrm{l}}$ (information matrices), two $m \times l$ matrices whose entries are letters from some alphabet.

For any $n$, a positive integer or $+\infty$, the two-person zero-sum game $\Gamma_{n}(p)$ is played as follows: Nature chooses one index $r$ in $K$ according to $p$. The maximizer (denoted by $P l_{\mathrm{l}}$ ) is informed in which element of $K^{\mathrm{I}}$ the index $r$ falls and the minimizer (denoted by $P l_{\mathrm{II}}$ ) is informed in which element of $K^{\mathrm{Il}}$ falls $r$. Then the players start repeating the following procedure $n$ times: At stage $t(t=1,2, \ldots) P l_{I}$ chooses $i_{t}$ in $\{1, \ldots, m\}$ and $P l_{I I}$ chooses $j_{t}$ in $\{1, \ldots, l\}$. The referee then informs $P l_{I}$ (resp. $P l_{\mathrm{II}}$ ) of the letter in the $\left(i_{t}, j_{t}\right)$ box of $H^{\mathrm{I}}$ (resp. $H^{\mathrm{I}}$ ). If $n$ is finite, $P l_{\mathrm{II}}$ pays $P l_{\mathrm{I}}$ the amount $(1 / n) \sum_{t=1}^{n} a_{i_{t}, j_{t}}^{r}$, and the value of $\Gamma_{n}(p)$ is denoted by $v_{n}(p)$. The description of the game is known to both players, and ends at this point.

In this model $K^{\mathrm{I}}$ (resp. $K^{\mathrm{II}}$ ) is the initial information of $P l_{\mathrm{l}}$ (resp. $P l_{\mathrm{II}}$ ) about the state of nature. It will be convenient to think of the elements of $K^{\mathrm{l}}$ (resp. $K^{\mathrm{II}}$ ) as the types of $P l_{1}$ (resp. $P l_{\mathrm{II}}$ ), we denote by $T^{\mathrm{l}}$ the set of types of $P l_{\mathrm{I}}$, i.e., $T^{\mathrm{I}}=\{1, \ldots, \mu\}$. The information matrix $H^{\mathrm{l}}$ (resp. $H^{\mathrm{II}}$ ) represents the information received by $P l_{1}$ (resp. $\left.P l_{11}\right)$ after a play of the game: The $(i, j)$ element of $H^{l}$ is the information "message" that goes to $P l_{\mathrm{I}}$ when he plays his $i$-th row and $P l_{\mathrm{II}}$ plays his $j$-th column ( $H^{\mathrm{Il}}$ has a similar meaning). It is very crucial to our proofs that this information is determined only by the players' moves and does not depend on the actual state of nature.

Aumann/Maschler [1967] and Stearns [1967] considered the subset of these games which have in addition the following two properties:

(i) The a-priori information of the players corresponds to what is called "the independent case" (to be defined later).

(ii) The information revealed during the play of the game consists of strategy choices of the players. This is referred to as "the standard information case".

The independent case is the special case in which $K$ can be arranged in a matrix such that the elements of $K^{\mathrm{I}}$ are the rows and those of $K^{\mathrm{II}}$ are the columns. In addition, the probability distribution $\pi$ on such $K$ is a product probability on $K^{\mathrm{I}} \times K^{\mathrm{II}}$ i.e. the probability of the $(\eta, \rho)$ element of $K$ is $\vec{p}_{\eta} q_{\rho}(\eta=1, \ldots, \mu ; \rho=1, \ldots, \nu)$ where $\left(p_{1}, \ldots, p_{\mu}\right)$ and $\left(q_{1}, \ldots, q_{\nu}\right)$ are two probability vectors. In other words, this is the case in which the type of $P l_{I}$ and the type of $P l_{I I}$ are chosen independently according to $\left(p_{1}, \ldots, p_{\mu}\right)$ and $\left(q_{1}, \ldots, q_{\nu}\right)$ respectively. It follows that in such a case $P l_{1}$ 's conditional probability on the types of $P l_{I I}$ is independent of his own type (and similarly for $P l_{\mathrm{II}}$ ). This is the motivation for the name "the independent case" given to this set of games.

The standard information case (condition (ii)) is formally the case in which $H^{\mathrm{I}}=H^{\mathrm{II}}=H$ where in each box of $H$ we write its coordinates $(i, j)$.

By passing from the Aumann-Maschler-Stearns model to the more general model presented here we thus dropped condition (i) and replaced condition (ii) by a much 
weaker condition, namely

(ii)* The players' information matrices $H^{\mathrm{I}}$ and $H^{\mathrm{II}}$ are independent of the state of nature.

In other words, the "message" received by the player after a certain play of the game is determined by the moves of the players at that play but not by the state of nature. (However it can be different to both players).

For the games satisfying (i) and (ii), Aumann/Maschler [1967] and Stearns [1967] proved that the Maxmin and the Minmax of the inf initely repeated game are Cav Vex $u$ and VexCav $u$ respectively, where $u(p)$ is the value of the one-shot game I II II I

with a-priori information $p$ in which the players are restricted to use non-revealing strategies (cfr. Definition 4) (Cav and Vex are operations of concavification and con-

vexification respectively, to be defined later). This result proves therefore a necessary and sufficient condition for the existence of value for the infinite game, for the family of games satisfying (i) and (ii), namely Cav Vex $u=$ Vex Cav $u$.

On the other hand Mertens/Zamir [1971] and Mertens [1971/72] proved that $\lim _{n \rightarrow \infty} \mathrm{v}_{n}$ exists (and in fact found this limit) for the larger family of games satisfying (ii)* (i.e. the games considered in this paper).

A natural question now arises whether it is possible to extend the Aumann-Maschler-Stearns condition for the existence of $v_{\infty}$ to the larger class of games for which $\lim \mathrm{v}_{n}$ is known. This paper gives an affirmative answer to this question.

Dropping condition (ii)* seems to be a quite difficult project and so far only very partial results exist [see e.g. Kohlberg/Zamir [1974] and Mertens/Zamir [1977]]. From these results it is already clear that both the condition for the existence of $v_{\infty}$ and the asymptotic value $\lim \mathrm{v}_{n}$ are quite different from the corresponding results for the games studied in this paper.

We would like to thank the referee for his useful suggestions concerning the presentation of the paper.

The result

Definition 1: $K^{\mathrm{l}}$ (resp. $K^{\mathrm{II}}$ ) is the $\sigma$-field generated by $K^{\mathrm{I}}$ (resp. $K^{\mathrm{Il}}$ ).

Definition 2: A function on the simplex of probabilities $p=\left(p^{1}, \ldots, p^{k}\right)$ is said to be concave with respect to I (for short, w.r.t.I) if for each $p$ it has a concave restriction to the subset $\Pi^{\mathrm{I}}(p)$ of the simplex, where

$$
\begin{gathered}
\Pi^{\mathrm{I}}(p)=\left\{\left(\alpha^{1} p^{1}, \ldots, \alpha^{k} p^{k}\right) \mid \alpha^{r} \geqslant 0, r=1, \ldots, k: \Sigma \alpha^{r} p^{r}=1\right. \\
\text { and } \left.\left(\alpha^{1}, \ldots, \alpha^{k}\right) \text { is } K^{\mathrm{I}} \text {-measurable }\right\},
\end{gathered}
$$

it is said to be convex with respect to II if for every $p$ it has a convex restriction to the subset $\Pi^{\mathrm{II}}(p)$, where

$$
\begin{gathered}
\mathrm{II}^{\mathrm{II}}(p)=\left\{\left(\beta^{1} p^{1}, \ldots, \beta^{k} p^{k}\right) \mid \beta^{r} \geqslant 0, r=1, \ldots, k: \Sigma \beta^{r} p^{r}=1\right. \\
\text { and } \left.\left(\beta^{1}, \ldots, \beta^{k}\right) \text { is } K^{\mathrm{II}} \text {-measurable }\right\},
\end{gathered}
$$

[cf. Mertens/Zamir, 1971, p. 42]. 
Remark: The set $\Pi^{\mathrm{I}}(p)$ (resp. $\left.\Pi^{\mathrm{II}}(p)\right)$ is loosely speaking, the set containing $p$ and on which $p\left(r \mid K^{\mathrm{l}}\right)$ (resp. $\left.p\left(r \mid K^{\mathrm{II}}\right)\right)$ is constant.

Definition 3: For a function $f$ on the simplex, the concavification of $f$ with respect to $I$, denoted by $\operatorname{Cav} f$, is the smallest function which majorizes $f$ and which is concave w.r.t.I. Similarly, the convexification of $f$ w.r.t.II, Vex $f$ is the largest function which minorizes $f$ and which is convex w.r.t.II, [cf.Mertens/Zamir, 1971, p. 42].

Definition 4: A one stage strategy of $P l_{1}$ is called non revealing if for each column of $H^{\mathrm{II}}$, the probability distribution induced on the letters of that column is independent of the state of nature $r \in K$. The set of non revealing one stage strategies of $P l_{\mathrm{I}}$ is denoted by $N R^{I}$. Similarly $N R^{I l}$ is the set of non revealing one stage strategies of $P l_{\text {II }}$, i.e., strategies such that for each row of $H^{\mathrm{I}}$, the probability distribution induced on the letters of that row does not depend on $r \in K,\left(N R^{\mathrm{I}}\right.$ and $N R^{\mathrm{II}}$ are obviously non empty).

Definition 5: $\Gamma_{N R}(p)$ is the one stage game in which $P l_{1}$ and $P l_{11}$ are restricted to strategies in $N R^{\mathrm{I}}$ and $N R^{\mathrm{II}}$ respectively. The value of $\Gamma_{N R}(p)$ is denoted by $u(p)$ (it is clearly continuous in $p$ ).

For any pair of strategies $\sigma$ and $\tau$ in $\Gamma_{\infty}(p)$ and for any positive integer $n$ we denote $\rho_{n}(\sigma, \tau)=E\left((1 / n) \sum_{t=1}^{n} a_{i_{t}, j_{t}}^{r}\right)$, where $E$ is the expectation with respect to the probability measure induced by $\sigma, \tau$ and $p$.

The result of this paper can be now stated as

\section{Theorem}

(a) For any strategy $\tau$ of $P l_{11}$ in $\Gamma_{\infty}(p)$ and any $\epsilon>0$ there is $N_{0}$ and a strategy $\sigma$ of $P l_{\mathrm{I}}$ such that $n>N_{0}$ implies $\rho_{n}(\sigma, \tau)>(\operatorname{Vex} \operatorname{Cav} u)(p)-\epsilon$;

b) There exists a strategy $\tau$ of $P l_{\mathrm{II}}$ such that for each $\epsilon>0$ there is $N$ such that

$$
\left.\rho_{n}(\sigma, \tau)<\underset{\text { II }}{\operatorname{Vex}} \operatorname{Cav} u\right)(p)+\epsilon \text { for all } n>N \text { and for any strategy } \sigma \text { of } P l_{[} \text {. }
$$

Remark: The content of the theorem is that Vex Cav $u$ is the Minmax of $\Gamma_{\infty}$ although we did not define the payoff for this game. Dually, Cav Vex is Maxmin.

In the independent and standard information case i.e. under conditions (i) and (ii), Aumann/Maschler [1967] proved part b) of our theorem and Stearns [1967] proved part a). To see the relation between two models, recall that the independent case is the case in which $K^{\mathrm{I}}=\{1, \ldots, \mu\}, K^{\mathrm{II}}=\{1, \ldots, \nu\}, K=K^{\mathrm{I}} \times K^{\mathrm{II}}$ and the probability distribution $\pi$ on $K$ is a product probability: $\pi=p \times q$ i.e. $\pi_{\eta \rho}=p_{\eta} q_{\rho}$, 
$\eta \in K^{\mathrm{l}}, \rho \in K^{\mathrm{ll}}, p=\left(p_{1}, \ldots, p_{\mu}\right)$ and $q=\left(q_{1}, \ldots, q_{\nu}\right)$ are probability distributions on $K^{\mathrm{I}}$ and $K^{\mathrm{II}}$ respectively (here we identify naturally an element $\eta$ of $K^{\mathrm{I}}$ with the subset $\{\eta\} \times K^{I I}$ of $K$, so that $K^{I}$ is a partition of $K$ as it should be, similarly for $K^{\text {II }}$.) It follows from the definition of $\Pi^{\mathrm{I}}(\pi)$ and $\Pi^{\mathrm{II}}(\pi)$ that $\Pi^{\mathrm{I}}(p \times q)=\left\{p^{\prime} \times q \mid p^{\prime}\right.$ is any probability distribution on $\left.K^{\mathrm{I}}\right\}$ and $\Pi^{\mathrm{II}}(p \times q)=\left\{p \times q^{\prime} \mid q^{\prime}\right.$ is any probability distribution on $K^{\mathrm{II}}$ \}. Consequently, the operation of concavification w.r.t.I. is actually the operation of concavifying for each fixed $q$ on the simplex of probability distributions $p$, and similarly for convexification. These are the operations Cav and Vex used by Aumann/Maschler and Stearns. To see what is $u-$ (the value of the one stage game with non revealing strategies) in the Aumann-Maschler-Stearns' case, observe that a general one-stage game strategy of $P l_{\mathrm{I}}$ is of the form $s=\left(s^{1}, \ldots, s^{\mu}\right)$ where for each $\eta \in K^{I}, s^{n}$ is a probability distribution on the set of rows $M=\{1, \ldots, m\}$ (this is the mixed strategy which is used by $P l_{\mathrm{I}}$ if he is of type $\eta$ ). Now in the standard information case (condition (ii)) definition 4 says that $s$ is non revealing if for each $j$ the probability distribution on $\{(i, j)\}$ induced by $s$ is independent of the state of nature, in particular independent of $P l_{I}$ 's type $\eta$. In other words the probability distribution on the rows $M$ has to be independent of $P l_{1}$ 's type i.e. $s^{1}=s^{2}=\ldots=s^{\mu}$. Similarly a non revealing strategy of $P l_{I I}$ is playing the same mixed strategy on the columns $L=\{1, \ldots, l\}$ independently of his type. This means that the one-stage game with non revealing strategies is equivalent to the matrix game whose payoff matrix is $\Delta(p, q)=\sum_{\eta \rho} p_{\eta} q_{\rho} A^{\eta \rho}$ und $u$ is thus the value of this game. This was precisely the definition of $u$ given by Aumann/Maschler and Stearns.

We turn now to the proof of the Theorem.

The proof of part $b$ ) is rather short and can be found at the end of the paper.

Outline of the proof of part a)

Given $P l_{\mathrm{II}}$ 's strategy, $P l_{1}$ can compute at every stage $t$ a posterior probability $p_{t}(r)$ on the true state of nature.

$P l_{\text {I }}$ will begin playing non revealing for some large number of times $N$, to exhaust a maximum of information from $P l_{\text {II }}$, i.e., such as to make $\sum_{r} E\left(p_{N}(r)-p(r)\right)^{2}$ as large as possible. Therefore, whatever $P l_{\mathrm{I}}$ plays after stage $N$, it will be as if $P l_{\mathrm{II}}$ was playing essentially non revealing after stage $N$.

Therefore, if we denote by $\tilde{p}_{N}$ the conditional probability distribution just before the $N+1$-th stage, $P l_{1}$ can guarantee $u\left(\tilde{p}_{N}\right)$ from stage $N+1$ on by playing in each stage his optimal strategy in $\Gamma_{N R}\left(\tilde{p}_{N}\right)$. Now we derive $\tilde{p}_{N}$ from $p_{N}$ (the conditional probability distribution on $K$ just after the $N$-th stage) by a procedure which we call "splitting". This is a very central element in the players' strategies and can be described as follows: $P l_{I}$ performs a lottery to choose an element $e$ from a fixed set $T^{I}$. The probability distribution on $T^{\mathrm{I}}$ may depend on $P l_{\mathrm{l}}$ 's type. Therefore hearing only the outcome $e$ (but not knowing according to which probability distribution it was chosen) one changes the prior probability distribution $p_{N}$ (on $K$ ) to some posterior probability distribution $\tilde{p}_{N}$. It is readily seen that $\tilde{p}_{N} \in \Pi^{\mathrm{I}}\left(p_{N}\right)$ and that $E\left(\tilde{p}_{N}\right)=p_{N}$. 
If after this lottery $P l_{\mathrm{I}}$ plays to guarantee $u\left(\tilde{p}_{N}\right)$, he guarantees with this procedure $E u\left(\tilde{p}_{N}\right)$. The maximum of this expression on the distribution of $\tilde{p}_{N}$ satisfying $\tilde{p}_{N} \in \Pi^{\mathrm{I}}\left(p_{N}\right)$ and $E\left(\tilde{p}_{N}\right)=p_{N}$, is exactly $(\underset{\mathrm{I}}{\operatorname{Cav}} u)\left(p_{N}\right)$. So $P l_{\mathrm{I}}$ can guarantee (Cav $u$ ) $\left(p_{N}\right)$ from stage $N+1$ on provided he can choose the type dependent lotteries so as to give the right probability distribution on $\tilde{p}_{N}$ which yields this maximum. It will be shown (Lemma 3 ) that in fact such lotteries can be found, hence $P l_{I}$ can guarantee $(\operatorname{Cav} u)\left(p_{N}\right)$ from stage $N+1$ on.

Finally, since $P l_{1}$ played non revealing up to stage $N$, we have $p_{N}\left(r \mid K^{I I}\right)=$ $=p\left(r \mid K^{I l}\right)$, and thus $E(\operatorname{Cav} u)\left(p_{N}\right) \geqslant(\underset{\text { II }}{\operatorname{Vex}} \operatorname{Cav} u)(p)$.

In Section 2 we give a sequence of lemmas and definitions that will be used in the proof of the theorem which will be given in Section 3.

\section{Preparations for the proof}

Lemma 1: Let $P$ be a positive measure on the product of two measurable spaces $(X, X) *(Y, Y)$ that has a density with respect to the product of it's marginals.

Then $E\|P(d y \mid x)-P(d y)\|=E\|P(d x \mid y)-P(d x)\|$.

Proof: By Fubini's theorem, both sides are equal to $\|P(d x, d y)-P(d x) \times P(d y)\|$.

Remark: This quantity is therefore a natural measure of independence between $X$ and $Y$.

Lemma 2: For any (Bochner-) integrable random variable $X$ with values in a Banach space $B$, and any $y \in B$

$$
E\|X-E(X)\| \leqslant 2 E\|X-y\|
$$

[cf. Mertens, p. 220].

Proof: $E\|X-E(X)\| \leqslant E\|X-y\|+\|E(X)-y\| \leqslant 2 E\|X-y\|$ by the triangle inequality and Jensen's inequality (using convexity of the norm).

The following was proved in Mertens/Zamir [1971, Lemma 2]:

Lemma 3: For each $p$ there exists a $K^{1}$-measurable transition probability $P(e \mid r)$ from $K$ to $T^{\mathrm{I}}$ such that if $p_{e}(r)=p(r \mid e), p_{e}=\left(p_{e}(1), \ldots, p_{e}(k)\right)$, then $u\left(p_{e}\right)=(\underset{I}{\operatorname{Cav}} u)\left(p_{e}\right)$ and $E u\left(p_{e}\right)=(\underset{I}{\operatorname{Cav} u})(p)$.

Lemma 4: Let $P_{\theta}$ be a transition probability from a probability space $(\Theta, \xi, P)$ to a measurable space $(\Omega, A)$, of which a finite collection $H$ of elements of $A$ is given; then 


$$
\inf _{P_{\theta}^{\prime} \in N R} E\left\|P_{\theta}-P_{\theta}^{\prime}\right\|_{1} \leqslant C \sum_{\alpha \in H} E\left|P_{\theta}(\alpha)-E\left(P_{\theta}(\alpha)\right)\right|
$$

for some constant $C$ depending only on the cardinality of the partition generated by $H$.

$$
P_{\theta}^{\prime} \in N R \text { means } P_{\theta}^{\prime}(\alpha) \text { is constant in } \theta \text { for any } \alpha \in H \text {. }
$$

The application of Lemma 4 to our context is the following: The set $\Theta$ is the set $K$ of states of nature, the space $\Omega$ is the set of rows (or columns) $M=\{1, \ldots, m\}$. A transition probability $P_{\Theta}$ is thus a one-stage strategy (of $P l_{\mathrm{I}}$ for instance). The left hand side of the above inequality is therefore a certain distance of the strategy $P_{\Theta}$ from the set of non revealing strategies. On the other hand the set $H$ stands in this context for the letters in the information matrix of the other player. A one-stage strategy $P_{\Theta}$ say of $P l_{\mathrm{I}}$ induces a probability distribution (given the column $j$ chosen) on the letters of $H^{\mathrm{II}}$ which depends usually on the state of nature, unless $P_{\Theta}$ is non revealing. The right hand side of the inequality above is a measure for how far is this probability on $H^{\text {II }}$ from being independent of $r$.

Summing up: Lemma 4 relates the distance of a one-stage strategy from the set of non revealing strategies (in the space of probability measures) to the extent to which the induced probability on the letters of $H^{\mathrm{II}}$ depend on the state of nature $r$.

Proof: This is Lemma 3 of Mertens [1971/72].

Lemma 5: The game $\Gamma_{n}(p)$ has essentially the same normal form (i.e., except for duplication of pure strategies) whether or not we assume for one or both of the players that he recalls, in addition to his initial information and his sequence of letters, his own past pure strategy choices.

Proof: This is Theorem 1 of Mertens [1971/72].

Remark: By virtue of this lemma, we will assume henceforth that no letter appears in two different rows of $H^{\mathrm{I}}$ or in two different columns of $H^{\mathrm{Il}}$. This modification will not change $N R^{\mathrm{I}}, N R^{\mathrm{II}}, \Gamma_{N R}(p)$ or $u(p)$. However it will enable us to identify letters with subsets of the pure strategy spaces of $P l_{\mathrm{I}}$ and $P l_{\mathrm{II}}$ - since any letter is now a product set of such a subset for $P l_{1}$ and of such a subset for $P l_{\mathrm{II}}$.

\section{Definition 6: [cf. Mertens, Lemma 2]}

$\underline{\Gamma}_{n}(p)$ is the $n$-times repeated game obtained by putting

1. $\underline{A}^{r}=\left(\begin{array}{c}A^{r} \\ -\underline{A}\end{array}\right)$, where $\underline{A}$ is the $m \times l$ matrix with constant entry $A$,

$$
A=\max _{i, j, r}\left|a_{i, j}^{r}\right|
$$

2. $\underline{H}^{\mathrm{I}}=\left(\begin{array}{c}S \\ H^{\mathrm{I}}\end{array}\right)$, where $S$ is the $m \times l$ matrix with constant entry $\alpha^{0}$, where $\alpha^{0}$ is a 
letter not appearing in $H^{\mathrm{I}}$.

3. $\underline{H}^{\mathrm{II}}=\left(\begin{array}{c}H^{\mathrm{II}} \\ \mathrm{I}\end{array}\right)$, where I is a $m \times l$ matrix all the letters of which are different among themselves and from the letters in $H^{\text {II }}$,

4. and by further restricting $P l_{I}$ to play each of his additional pure strategies (i.e., those numbered from $m+1$ to $2 m$ ) which probability $\delta / m$.

We will use letters $\alpha$ (with subscripts) for the elements of $\underline{H}^{\mathrm{I}}$ and letters $\beta$ (with subscripts) for the elements of $\underline{H}^{\mathrm{II}}$.

Remark that, as noticed after Lemma 5 , the letters and $\alpha$ and $\beta$ can all be identified with rectangles in the product set $L \times M=\{1, \ldots, l\} \times\{1, \ldots m, m+1, \ldots, 2 m\}$.

Lemma 6: Every strategy of $P l_{\mathrm{I}}$ in $\Gamma_{n}(p)$ is dominated by a strategy of $P l_{\mathrm{I}}$ in $\Gamma_{n}(p)$ [cf. Mertens, Lemma 2].

Denote by $\mathcal{H}^{\mathrm{I}}\left(\right.$ resp. $\left.\mathcal{H}^{\mathrm{II}}\right)$ the field on $L \times \underline{M}$ generated by $\underline{H}^{\mathrm{I}}$ (resp. $\underline{H}^{\mathrm{II}}$ ), and remark that, by Definition $6, \mathcal{H}^{\mathrm{I}} \subseteq \mathcal{H}^{\mathrm{II}}$.

Let $\Omega=K \times\left[L \times \underline{M} \times T^{1}\right]^{\infty}$. The $t$-th factor space $L, \underline{M}$ and $T^{\mathrm{I}}$ will be denoted by $L_{t}, \underline{M}_{t}$ and $T_{t}^{\mathrm{I}}$ respectively. Let $\varepsilon_{t}^{\mathrm{II}}$ be the field on $\Omega$ generated by $K^{\mathrm{II}}$ and the fields $\mathcal{T}^{\mathrm{II}}$ on the fisrt $t-1$ factor spaces $L \times \underline{M}$. \& is the field generated by the first $(t-1)$ factor spaces $T^{\mathrm{I}}$ and by the fields $\mathcal{H}^{\mathrm{I}}$ on the first $(t-1)$ factor spaces $L \times \underline{M}$. $\tilde{\mathscr{E}}_{t}$ is generated by $\varepsilon_{t}$ and $T_{t}^{l}$.

$A \vee B$ denotes the $\sigma$-field generated by the $\sigma$-fields $A$ and $B$. Also let

$$
\varepsilon_{t}^{I}=\varepsilon_{t} \vee K^{I}, \widetilde{\varepsilon}_{t}^{I}=\widetilde{\mathscr{\varepsilon}}_{t} \vee K^{\mathrm{I}}, \tilde{\mathbb{\varepsilon}}_{t}^{\mathrm{II}}=\tilde{\mathbb{\varepsilon}}_{t} \vee K^{\mathrm{II}} .
$$

$K$ will be the field generated by the first factor space, $K$.

Denote by $G_{t}$ the field generated by $K$ and the first $(t-1)$ factor spaces $L \times M \times T^{l}$, and by $\widetilde{G}_{t}$ the field generated by $G_{t}$ and $T_{t}^{l}$.

By virtue of Lemma 5 , we may assume that $P l_{I I}$ uses a behavioral strategy, i.e., at every stage $t$ he uses a transition probability $\tau_{t}$ from $\left(\Omega, \mathbb{E}_{t}^{\mathrm{II}}\right)$ to the $t$-th factor space $L$.

The only strategies for $P l_{\mathrm{I}}$ we will consider will be of the following type: at each stage $t$, use first a transition probability from $\left(\Omega, \varepsilon_{t}^{\mathrm{I}}\right)$ to $T_{t}^{\mathrm{I}}$, then a $\widetilde{\mathrm{E}}_{t}$-measurable function from $\Omega$ to $N R^{I}$, which selects a point in $\underline{M}_{t}$.

Given such a pair of strategies $\sigma$ and $\tau$ for both players, and the probability distribution $p=\left(p^{1}, \ldots, p^{k}\right)$ on $K$ of the initial choice of nature, the probability $P_{\sigma, \tau}$ on $\left(\Omega, G_{\infty}\right)$ is completely defined by the following requirements:

a) The conditional distribution on $T_{t}^{\mathrm{I}}$ given $G_{t}$ is $\&_{t}^{\mathrm{I}}$-measurable, and is given by $P l_{I}$ 's strategy (thus: $T_{t}^{1}$ and $G_{t}$ are conditionally independent given $\varepsilon_{t}^{1}$ ).

b) $L_{t}$ and $M_{t}$ are conditionally independent given $\widetilde{G}_{t}$.

c) The conditional distribution on $L_{t}$ given $\widetilde{G}_{t}$ is ${ }_{E_{t}}^{I I}$-measurable, and is given by $P l_{I I}$ 's strategy (thus: $L_{t}$ and $\widetilde{G}_{t}$ are conditionally independent given $\mathscr{Q}_{t}^{\mathrm{II}}$ ).

d) The conditional distribution on $\underline{M}_{t}$ given $\widetilde{G}_{t}$ is $\widetilde{\mathscr{E}}_{t}^{\mathrm{I}}$-measurable, and is given by $P l_{\mathrm{I}}$ 's strategy (thus: $\underline{M}_{t}$ and $\widetilde{G}_{t}$ are conditionally independent given $\widetilde{\mathscr{G}}_{t}^{I}$ ). 
e) For any letter $\beta \in H^{\mathrm{II}}$, considered as a subset of $\underline{M}_{t}$, the occurence of $\beta$ and $\widetilde{G}_{t}$ are conditionally independent given $\widetilde{\mathscr{E}}_{t}$ (i.e., the conditional probability of $\beta$ given $\tilde{G}_{t}$ is $\tilde{\mathscr{E}}_{t} \cdot$ measurable).

We will write $p_{t}(r)$ for $P\left(r \mid \varepsilon_{t}\right)$ and $\tilde{p}_{t}(r)$ for $P\left(r \mid \tilde{\varepsilon}_{t}\right)$.

We now prove a basic property of this probability space [Lemma 6 of Mertens], with a proof which is somewhat simpler and probably more convincing than the original one.

Lemma 7: $\varepsilon_{t+1}^{\mathrm{II}}$ and $K$ are conditionally independent given $\widetilde{\varepsilon}_{t}^{\mathrm{II}}$.

Corollary 8: $p_{t+1}\left(r \mid K^{\mathrm{II}}\right)=P\left(r \mid \alpha_{1} \ldots \alpha_{t}, e_{1} \ldots e_{t}, \rho\right)=P\left(r \mid \beta_{1} \ldots \beta_{t}, e_{1} \ldots e_{t}, \rho\right)=$ $=P\left(r \mid \beta_{1} \ldots \beta_{t-1}, e_{1} \ldots e_{t}, \rho\right)=P\left(r \mid \alpha_{1} \ldots \alpha_{t-1}, e_{1} \ldots e_{t}, \rho\right)=\tilde{p}_{t}\left(r \mid K^{\mathrm{II}}\right)$.

Here $\rho$ stands for an element of $K^{\mathrm{II}}$ and $e_{i}, i=1, \ldots, t$ are elements of $T^{\mathrm{I}}$.

\section{Proof of Corollary 8:}

- The first and last equalities are definitions.

- The equality of the third and the next to last term is equivalent to Lemma 7.

- The conditioning $\sigma$-fields in the second and in the fourth term are intermediate between those in the third and the next to last term.

Proof of Lemma 7: Proceeds by induction on $t$. Recall that the letters $\alpha$ may be considered as forming a partition of the letters $\beta$. We assume $r \in \rho \in K^{\mathrm{II}}$. By induction hypothesis we have (cf. Corollary 8):

$$
P\left(r \mid \beta_{1} \ldots \beta_{t}, e_{1} \ldots e_{t}, \rho\right)=P\left(r \mid \alpha_{1} \ldots \alpha_{t}, e_{1} \ldots e_{t}, \rho\right)
$$

this holds obviously also for $t=0$. On the other hand we have by (a):

$$
P\left(e_{t+1} \mid \beta_{1} \ldots \beta_{t}, e_{1} \ldots e_{t}, r\right)=P\left(e_{t+1} \mid \alpha_{1} \ldots \alpha_{t}, e_{1} \ldots e_{t}, r\right)
$$

These two relations imply:

$$
P\left(e_{t+1}, r \mid \beta_{1} \ldots \beta_{t}, e_{1} \ldots e_{t}, \rho\right)=P\left(e_{t+1}, r \mid \alpha_{1} \ldots \alpha_{t}, e_{1} \ldots e_{t}, \rho\right)
$$

and thus $P\left(r \mid \beta_{1} \ldots \beta_{t}, e_{1} \ldots e_{t+1}, \rho\right)=P\left(r \mid \alpha_{1} \ldots \alpha_{t}, e_{1} \ldots e_{t+1}, \rho\right)$, which is the conditional independence of $\mathscr{E}_{t+1}^{\mathrm{II}}$ and $K$ given $\widetilde{\mathscr{E}}_{t+1}^{\mathrm{II}}$ (cf. Corollary 9 ), or, in other words,

$$
P\left(\beta_{1} \ldots \beta_{t} \mid \alpha_{1} \ldots \alpha_{t}, e_{1} \ldots e_{t+1}, r\right)=P\left(\beta_{1} \ldots \beta_{t} \mid \alpha_{1} \ldots \alpha_{t}, e_{1} \ldots e_{t+1}, \rho\right)\left({ }^{* *}\right)
$$

By (b) (c) and (e) we have that

$$
p\left(\beta_{t+1} \mid \widetilde{G}_{t+1}\right)=P\left(j \in \beta_{t+1} \mid \beta_{1} \ldots \beta_{t}, \rho\right) P\left(i \in \beta_{t+1} \mid \alpha_{1} \ldots \alpha_{t}, e_{1} \ldots e_{t+1}\right),
$$


and thus

$$
P\left(\beta_{t+1} \mid \widetilde{G}_{t+1}\right)=P\left(\beta_{t+1} \mid \beta_{1} \ldots \beta_{t}, e_{1} \ldots e_{t+1}, \rho\right)
$$

a fortiori

$$
P\left(\beta_{t+1} \mid \beta_{1} \ldots \beta_{t}, e_{1} \ldots e_{t+1}, r\right)=P\left(\beta_{t+1} \mid \beta_{1} \ldots \beta_{t}, e_{1} \ldots e_{t+1}, \rho\right)
$$

Together with formula $(* *)$, this implies

$$
P\left(\beta_{1} \ldots \beta_{t+1} \mid \alpha_{1} \ldots \alpha_{t}, e_{1} \ldots e_{t+1}, r\right)=P\left(\beta_{1} \ldots \beta_{t+1} \mid \alpha_{1} \ldots \alpha_{t}, e_{1} \ldots e_{t+1}, \rho\right)
$$

which is the conditional independence of $\mathbb{E}_{t+2}^{I I}$ and $K$ given $\tilde{\mathscr{E}}_{t+1}^{I I}$.

Corollary 9: $\mathscr{\&}_{t}^{I I}$ and $\left(K \vee \widetilde{\varepsilon}_{t}\right)$ are conditionally independent given $\varepsilon_{t} \vee K^{I I}$.

Proof: This is formula (*).

Definition 7: For any strategy $\sigma$ of $P l_{\mathrm{I}}$, for any time $t$, and for any $e \in T^{\mathrm{I}}$, denote by $\sigma_{t, e}$ the strategy (i.e., set of transition probabilities) of $P l_{\mathrm{I}}$ that coincides with $\sigma$ except that at time $t, P\left(e_{t}=e \mid G_{t}\right)=1$.

Lemma 10: For any strategies $\sigma$ of $P l_{\mathrm{I}}$ and $\tau$ of $P l_{\mathrm{II}}$, for any time $t$ and $e \in T^{\mathrm{I}}$, the conditional distributions given $\widetilde{\mathbb{E}}_{t}^{\mathrm{I}}$ induced by $P_{\sigma, \tau}$ and $P_{\sigma_{t, e}, \tau}$ on $G_{\infty}$ coincide on
$\left\{e_{t}=e\right\}$.

Proof: We have to show that, for any $n \geqslant t$, the following probability is the same whether $P$ stands for $P_{\sigma, \tau}$ or $P_{\sigma, e}, \tau$ (we assume $r \in \rho \in K^{\mathrm{I}}$ ):

$$
\begin{array}{r}
P\left(r, e_{1}, i_{1}, j_{1}, \ldots, e_{t-1}, i_{t-1}, j_{t-1}, e_{t}, i_{t}, j_{t}, \ldots, e_{n}, i_{n}, j_{n}, e_{n+1} \mid \rho\right. \\
\left.e_{1}, \alpha_{1}, e_{2}, \alpha_{2}, \ldots, e_{t-1}, \alpha_{t-1} ; e_{t}\right) .
\end{array}
$$

Using inductively a) and b), c), d), this statement can be reduced to the case where $n=t-1$, i.e. to:

$$
P\left(r ; e_{1}, i_{1}, j_{1}, e_{1}, \ldots, e_{t-1}, i_{t-1}, j_{t-1}, e_{t} \mid \rho ; e_{1}, \alpha_{1}, e_{2}, \alpha_{2}, \ldots, e_{t-1}, \alpha_{t-1}, e_{t}\right)
$$

which equals

$$
P\left(r ; e_{1}, i_{1}, j_{1}, \ldots, e_{t-1}, i_{t-1}, j_{t-1} \mid \rho ; e_{1}, \alpha_{1}, \ldots, e_{t-1}, \alpha_{t-1}, e_{t}\right)
$$

which, by a), equals

$$
P\left(r ; e_{1}, i_{1}, j_{1}, \ldots, e_{t-1}, i_{t-1}, j_{t-1} \mid \rho ; e_{1}, \alpha_{1}, \ldots, e_{t-1}, \alpha_{t-1}\right)
$$


The result now follows from the fact that $P_{\sigma, \tau}$ and $P_{\sigma, e^{, \tau}}$ coincide on $G_{t}$.

Lemma 11: $E\left(\mid p_{t+1}(r)-\tilde{p}_{t}(r) \| \widetilde{\mathscr{\varepsilon}}_{t}\right)=\tilde{p}_{t}(r) \frac{\delta}{m} \sum_{\alpha \in H} \mid P\left(j_{t} \in \alpha \mid \widetilde{\mathscr{\varepsilon}}_{t}, r\right)-$ $-P\left(j_{t} \in \alpha \mid \widetilde{\varepsilon}_{t}\right) \mid$

Proof: cf. Mertens [1971/72, p. 224, formula (3)].

Definition 8: Let $\omega$ stand for a typical point in $\widetilde{\varepsilon}_{t}$, and denote $P\left(j_{t}=j \mid \omega, r\right)$ by $\vec{\tau}^{r}(\omega)(j)$.

By c) and Corollary $9, \bar{\tau}^{r}$ is $K^{\mathrm{II}}$-measurable in $r$.

\section{Lemma 12:}

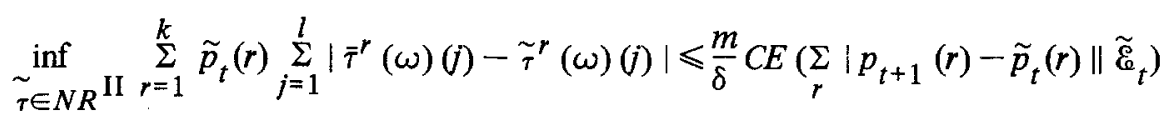

where $C$ is a constant that depends only on the cardinality of the partition of $L$ generated by the letters $\alpha$ of $H^{\mathrm{I}}$.

Proof: Follows from Lemmas 4 and 11.

Definiton 9:

a) $N R_{\infty}^{\mathrm{I}}$ is the set of strategies of $P l_{\mathrm{I}}$ such that, for every $t$, and for every $e \in T^{\mathrm{I}}$, $P\left(e_{t}=e\right) \in\{0,1\}$.

b) Given a strategy $\tau$ of $P l_{\mathrm{II}}$ and $\epsilon>0$, define $\sigma_{0} \in N R_{\infty}^{\mathrm{I}}$ and $N$ by

$$
E_{\sigma_{0}, r^{\prime}} \sum_{r=1}^{k} \sum_{t=1}^{N-1}\left(p_{t+1}(r)-p_{t}(r)\right)^{2}>\operatorname{Sup}_{\sigma \in N R_{\infty}} E_{\sigma, \tau} \sum_{r=1}^{k} \sum_{t=1}^{\infty}\left(p_{t+1}(r)-p_{t}(r)\right)^{2}-\epsilon .
$$

Lemma 13: For any strategy $\sigma$ in $N R_{\infty}^{\mathrm{I}}$, that coincides with $\sigma_{0}$ up to stage $N$, (i.e., on $G_{N}$ ) and for any $n \geqslant N$, we have

$$
E \sum_{r=1}^{k}\left|P\left(r \mid \widetilde{\varepsilon}_{n}\right)-P\left(r \mid \widetilde{\mathscr{\varepsilon}}_{N}\right)\right| \leqslant \sqrt{k \epsilon}
$$

Proof: Follows immediately from Hölder's inequality and the definitions of $N R_{\infty}^{\mathrm{I}}, \sigma_{0}$ and $N$.

Lemma 14: Consider any strategy $\sigma$ of $P l_{\mathrm{I}}$, that coincides with $\sigma_{0}$ up to stage $N$, and such that, for all $\tau$, for all $e \in T^{1}$, and for $t \neq N, P\left(e_{t}=e\right) \in\{0,1\}$. Then, for any $n \geqslant N$

$$
E\left(\underset{r}{\sum}\left|\tilde{p}_{n}(r)-\tilde{p}_{N}(r)\right|\right) \leqslant 2 \mu \sqrt{k \epsilon} .
$$


Proof: By Lemma 1, applied conditionally to $\widetilde{\mathscr{E}}_{N}$, we have

$$
\begin{aligned}
& E\left(\sum_{r} \mid P\left(r \mid \widetilde{\varepsilon}_{n}\right)-P\left(r \mid \widetilde{\varepsilon}_{N}\right) \| \widetilde{\mathscr{\varepsilon}}_{N}\right) \\
& \quad=\sum_{r} P\left(r \mid \widetilde{\mathscr{E}}_{N}\right)\left\|P\left(\cdot \mid \widetilde{\varepsilon}_{N}, r\right)-\sum_{r} P\left(r \mid \widetilde{\varepsilon}_{N}\right) P\left(\cdot \mid \widetilde{\varepsilon}_{N}, r\right)\right\|_{\widetilde{\varepsilon}_{n}} .
\end{aligned}
$$

By Lemma 2, applied conditionally to $\widetilde{\varepsilon}_{N}$, we get therefore

$$
\left.E \underset{r}{\Sigma} \mid \tilde{p}_{n}(r)-\tilde{p}_{N}(r) \| \widetilde{\varepsilon}_{N}\right) \leqslant 2 \sum_{r} \tilde{p}_{N}(r)\left\|P\left(\cdot \mid \widetilde{\varepsilon}_{N}, r\right)-\sum_{r} P\left(r \mid \varepsilon_{N}\right) P\left(\cdot \mid \widetilde{\varepsilon}_{N}, r\right)\right\|_{\widetilde{\varepsilon}_{N}} .
$$

By Lemma 10 , we have that

$$
\begin{aligned}
X(r, \omega, e) & =\left\|P_{\sigma, \tau}\left(\cdot \mid \widetilde{\mathscr{E}}_{N}, r\right)-\sum_{r} P\left(r \mid \mathscr{E}_{N}\right) P_{\sigma, \tau}\left(\cdot \mid \widetilde{\mathscr{E}}_{N}, r\right)\right\|_{\widetilde{\mathscr{E}}_{n}} \\
& =\left\|P_{\sigma_{N, e}, \tau}\left(\cdot \mid \widetilde{\mathscr{E}}_{N}, r\right)-\sum_{r} P_{\sigma_{N, e^{, \tau}}}\left(r \mid \widetilde{\mathscr{E}}_{N}\right) P_{\sigma_{N, e}, \tau}\left(\cdot \mid \widetilde{\mathscr{E}}_{N}, r\right)\right\|_{\widetilde{\mathscr{G}}_{n}}
\end{aligned}
$$

(where $\omega$ stands for a typical point of $\mathscr{E}_{N}$, and $e$ for a point in $T_{N}^{!}$).

Let now $\sum_{r} P_{\sigma_{N, e^{,}}}\left(r \mid \&_{N}\right) X(r, \omega, e)=Y(e, \omega)$. Then

$$
\begin{aligned}
& E\left(\sum_{r} \mid \tilde{p}_{n}(r)-\tilde{p}_{N}(r) \| \varepsilon_{N}\right) \leqslant 2 \sum_{e} \sum_{r} P(r, e \mid \omega) X(r, \omega, e) \leqslant \\
& \leqslant 2 \sum_{r} P(r \mid \omega) \max _{e} X(r, \omega, e) \leqslant 2 \sum_{r} P(r \mid \omega) \sum_{e} X(r, \omega, e)=2 \sum_{e=1}^{\mu} Y(e, \omega) .
\end{aligned}
$$

But by Lemma's 1 and $13, Y(e, \omega)$ has expectation $\leqslant \sqrt{k \epsilon}$ for every $e$, and thus

$$
E\left[\underset{r}{\sum}\left|\tilde{p}_{n}(r)-\tilde{p}_{N}(r)\right|\right] \leqslant 2 \mu \sqrt{k \epsilon}
$$

\section{Proof of the Theorem}

For any given strategy $\tau$ of $P l_{\mathrm{II}}$ and $\epsilon>0$ define $\sigma_{0}$ and $N$ as in b) of Definition 9 and consider the strategy $\sigma$ of $P l_{1}$ defined as follows:

(i) Play $\sigma_{0}$ up to stage $N$.

(ii) Use the transition probability described in Lemma 3, with $p=p_{N}$, to choose

(iii) From stage $N$ on play at every stage independently an optimal strategy in $\Gamma_{N R}\left(\tilde{p}_{N}\right)$.

For $t \geqslant N$ denote by $H_{t}$ the conditional expected payoff at stage $t$ given $\widetilde{\varepsilon}_{t}$. For the given $\tau$ and the above described $\sigma$ we have: 


$$
\begin{aligned}
& H_{t}=E\left(a_{i_{t}, j_{t}}^{r} \mid \widetilde{\varepsilon}_{t}\right)=E\left[E\left(\underline{g} \underline{A} \tau \tilde{\varepsilon}_{t} \vee K\right) \mid \widetilde{\varepsilon}_{t}\right]=E\left[\underline{g} \underline{A} E\left(\tau \mid \widetilde{\varepsilon}_{t} \vee K\right) \mid \widetilde{\varepsilon}_{t}\right] \\
& =E\left(\sigma \underline{A} \bar{\tau} \mid \widetilde{\complement}_{t}\right) \text {, by Definition } 8 \text {. }
\end{aligned}
$$

Let $\omega$ be a typical point in $\widetilde{\mathscr{E}}_{t}$, then $\underline{\sigma}=\underline{\sigma}^{r}(\omega)$ is $K^{\mathrm{I}}$-measurable in $r$ and $\bar{\tau}=\bar{\tau}^{r}(\omega)$ is $K^{\mathrm{II}}$-measurable in $r$, and so:

$$
H_{t}(\omega)=\sum_{r=1}^{k} \tilde{p}_{t}(r) \underline{\sigma}^{r}(\omega) \underline{A}^{r} \bar{\tau}^{r}(\omega) \text { with } \tilde{p}_{t}(r)=p(r \mid \omega)
$$

from which:

$$
H_{t}(\omega) \geqslant \sum_{r=1}^{k} \tilde{p}_{N}(r) \underline{\sigma}^{r}(\omega) \underline{A}^{r} \vec{\tau}^{r}(\omega)-A \sum_{r=1}^{k}\left|\tilde{p}_{N}(r)-\tilde{p}_{t}(r)\right| .
$$

If $\tilde{\tau}^{r}(\omega)$ is in $N R^{I I}$ then:

$$
\begin{gathered}
H_{t}(\omega) \geqslant(1-\delta) \sum_{r=1}^{k} \tilde{p}_{N}(r) \sigma^{r}(\omega) A^{r} \tilde{\tau}^{r}(\omega) \\
-(1-\delta) A \sum_{r=1}^{k} \tilde{p}_{t}(r) \sum_{j=1}^{l}\left|\bar{\tau}^{r}(\omega)(j)-\tilde{\tau}^{r}(\omega)(j)\right|-A \sum_{r=1}^{k}\left|\tilde{p}_{N}(r)-\tilde{p}_{t}(r)\right|-\delta A .
\end{gathered}
$$

Since $\sigma^{r}(\omega)$ is optimal in $\Gamma_{N R}\left(\tilde{p}_{N}\right)$ we get:

$$
\begin{aligned}
H_{t}(\omega) \geqslant u\left(\tilde{p}_{N}\right)-A \sum_{r=1}^{k} \tilde{p}_{t}(r) \sum_{j=1}^{l} \mid & \tilde{\tau}^{r}(\omega)(j)-\tilde{\tau}^{r}(\omega)(j) \mid- \\
& -A \sum_{r=1}^{k}\left|\tilde{p}_{N}(r)-\tilde{p}_{t}(r)\right|-2 \delta A .
\end{aligned}
$$

By Lemma 12

$$
\begin{gathered}
H_{t}(\omega) \geqslant u\left(\tilde{p}_{N}\right)-\frac{A C m}{\delta} \sum_{r=1}^{k} E\left(\mid p_{t+1}(r)-\tilde{p}_{t}(r) \| \widetilde{\varepsilon}_{t}\right)-A \sum_{r=1}^{k} \mid \tilde{p}_{N}(r)- \\
-\tilde{p}_{t}(r) \mid-2 \delta A .
\end{gathered}
$$

Remark that $E\left(u\left(\widetilde{p}_{N}\right) \mid \mathscr{E}_{N}\right)=(\underset{I}{\operatorname{Cav}} u)\left(p_{N}\right) \geqslant(\underset{\text { II }}{\operatorname{Vex}} \operatorname{Iav} u)\left(p_{N}\right)$, therefore it follows from Jensen's inequality [cf. Mertens, the argument at the end of page 224] that:

$$
E\left(u\left(\tilde{p}_{N}\right)\right) \geqslant(\underset{\text { II }}{\operatorname{Vex}} \operatorname{Cav} u)(p) .
$$

Thus we get for the expectation of $H_{t}$, using Lemma 14: 


$$
\begin{aligned}
& \left.E\left(H_{t}(\omega)\right) \geqslant \underset{\text { II }}{\operatorname{Vex}} \operatorname{Cav} u\right)(p)-\frac{A C m}{\delta} \sum_{r=1}^{k} E\left|p_{t+1}(r)-\tilde{p}_{t}(r)\right|-2 A \mu \sqrt{k} \bar{\epsilon}- \\
& -2 A \delta \text {. }
\end{aligned}
$$

Summing on $t$ from $N$ to $N+n$, and using

$$
\sum_{r=1}^{k} \frac{1}{n} \sum_{t=N}^{n+N} E\left|p_{t+1}(r)-\tilde{p}_{t}(r)\right| \leqslant \sqrt{\frac{k-1}{n}}
$$

(by Hölder's inequality), we get:

$$
\rho_{N+n}(\sigma, \tau) \geqslant-\frac{2 A N}{n+N}+(\operatorname{Vex} \operatorname{Cav} u)(p)-2 A \delta-2 A \mu \sqrt{k \epsilon}-\frac{A C m}{\delta} \sqrt{\frac{k-1}{n}}
$$

Finally, for each $\tilde{\epsilon}$ we may choose $N_{0}$ big enough, $\epsilon$ and $\delta$ small enough as to have

$$
\rho_{n}(\sigma, \tau)>(\underset{\text { II }}{\operatorname{Cav}} u)(p)-\tilde{\epsilon} \text { whenever } n>N_{0}
$$

This finishes the proof of Part (a).

The proof of the second part of the theorem is directly inspired from that given by. Aumann/Maschler [1967]. We show it is even true in the game $\bar{\Gamma}_{\infty}$ (def. dual of def. 6).

First, notice that the Aumann/Maschler result holds also if we allow the entries of the information matrices $H^{\mathrm{I}}$ and $H^{\mathrm{II}}$ to be not just letters from a finite alphabet $H$ but rather distributions on $H$. Observe now that the set $N R^{\text {II }}$ is a polyhedral convex set which is therefore the convex hull of a finite set of extreme non revealing strategies. Let $\tau=\left(\tau^{1}, \ldots, \tau^{k}\right)$ be such an extreme point of $N R^{I I}$. For each $r \in K$ let us add to the payoff matrix $\bar{A}^{r}$ an additional column which is the convex combination of the columns $\{1, \ldots, l\}$ according to the mixed strategy $\tau^{r}$. Let us add to $\bar{H}^{\mathrm{I}}$ a corresponding additional column whose $i$-th element is the probability distribution on the letters of $\bar{H}^{I}$ induced by $\tau$ when $P l_{\mathrm{I}}$ is playing the $i$-th row, (since $\tau \in N R^{I I}$, this distribution is independent of $r$.) Similarly, in $\bar{H}^{\text {II }}$ a column of $\beta_{0}$ is added. Now, adding this column clearly has not changed the game, (since the additional column is a strategy that was available to $P l_{\mathrm{II}}$ in the original game too). However, the non revealing strategy $\tau$ is now represented as a constant strategy (independent of $r$.) Thus adding in this way one column for each extreme point of $N R^{\text {II }}$ we do not change to the game but represent all strategies in $N R^{\text {II }}$ as constant strategies. In other words if $P l_{\text {II }}$ restricts himself to strategies in $N R^{\text {II }}$, we may as well assume that he plays independently of $r$ (namely mixtures of the added columns only.)

Thus if $P l_{\mathrm{II}}$ restricts himself to strategies in $N R^{\mathrm{II}}$ and if for each $\rho \in T^{\mathrm{I}}$ we let $q^{\rho}=\sum_{r \in \rho} p^{r} ; A^{\rho}=\frac{1}{q^{\rho}} \sum_{r \in \rho} p^{r} A^{r}$ we obtain a game of incomplete information on one side $\left(P l_{11}\right)$ with $T^{\mathrm{I}}$ as the set of states of nature and $q$ as the probability distribution on it. By Aumann/Maschler [1968], the value of this game is Cav v, where v( $q)$ is the value 
of the game in which $P l_{\mathrm{I}}$ plays non revealing.

Now by our construction $\mathrm{v}(q)=u(p)$ and $\mathrm{Cav}=\mathrm{Cav}$, so we know by Aumann/ Maschler [1968] that $P l_{\text {II }}$ can guarantee $(\mathrm{Cav} u)(p)$. Therefore by Lemma 3 (or rather its dual for $P l_{\text {II }}$ ) he can guarantee (Vex Cav $u$ ) (p) using that lottery before the first stage, and from then on guaranteeing $(\operatorname{Cav} u)(p)$, where $p$ is the posterior on $K$ given the outcome of that lottery.

This completes the proof of the theorem.

\section{References}

Aumann, R.J., and M. Maschler: Repeated Games with Incomplete Information. A Survey of Recent Results. Report to the U.S. Arms Control and Disarmament Agency. Washington D.C. Final Report on Control ACDA/ST-116, prepared by Mathematica, Princeton, N.J., September 1967, Chapter III, 287-403.

-: Repeated Games with Incomplete Information. The Zero-Sum Extensive Case. Report to the U.S. Arms Control and Disarmament Agency. Washington D.C. Final Report on Contract ACDA/ST-143, prepared by Mathematica, Princeton, N.J., November 1968, Chapter II, 25-108.

Kohlberg, E., and $S$. Zamir: Repeated Games of Incomplete Information: The symmetric Case. The Annals of Statistics 2, 1974, 1040-1041.

Mertens, J.F.: The value of Two-Person Zero-Sum Repeated Games: The Extensive Case, International Journal of Game Theory 1, 1971/72, 217-227.

Mertens, J.F., and S. Zamir: The Value of Two-Person Zero-Sum Repeated Games with Lack of Information on Both Sides. International Journal of Game Theory 1, 1971, 39-64.

-: On a Repeated Game Without a Recursive Structure. International Journal of Game Theory 5 , $1977,173-182$.

Stearns, R.E. : A Formal Information Concept for Games with Incomplete Information. Report to the U.S. Arms Control and Disarmament Agency. Washington D.C. Final Report on Contract ACDA/ST-116, prepared by Mathematica, Princeton, N.J., September 1967, Chapter IV, $405-433$.

Received November, 1977

(revised version September, 1979) 